\title{
ON THE EXISTENCE OF DEAD CORES FOR DEGENERATE LOTKA-VOLTERRA MODELS
}

\author{
Manuel DELGADO and Antonio SUÁREZ \\ Dpto. de Ecuaciones Diferenciales y Análisis Numérico, Facultad de Matemáticas, \\ C/ Tarfia s/n, Universidad de Sevilla, 41012-Sevilla, Spain. \\ e-mail: delgado@numer.us.es and suarez@numer.us.es
}

\begin{abstract}
In this work we study the existence, uniqueness and qualitative properties of nonnegative solutions of the Lotka-Volterra models with nonlinear diffusion under homogeneous Dirichlet boundary conditions. We consider the three typical interactions: prey-predator, competition and symbiosis. Unlike the linear diffusion models, nontrivial nonnegative solutions can exist which are not strictly positive. Sufficient conditions in terms of the coefficients involved in the setting of the models are given assuring that one species (or both) does not survive on a set of its habitat (called "dead core") of positive measure.
\end{abstract}

AMS Classification Codes: 35B50, 35B99, 35J55, 35K57, 92D25.

Keywords and phrases: degenerate Lotka-Volterra models, coexistence states, dead core set. 


\section{Introduction}

In this paper we are interested in nonnegative solutions of the following system

$$
\begin{cases}-d_{1} \Delta U^{m}=U(A-B U \pm C V) & \text { in } \Omega, \\ -d_{2} \Delta V^{n}=V(D-E V \pm F U) & \text { in } \Omega, \\ U=V=0 & \text { on } \partial \Omega\end{cases}
$$

where $\Omega$ is a bounded domain of $\mathbb{R}^{N}, N \geq 1$, with a smooth boundary $\partial \Omega$ and $m, n, d_{1}, d_{2}, B$, $C, E, F$ are positive constants with $m, n \geq 1$ and $A, D \in \mathbb{R}$. The problem (1) models the interactions between two species, with population densities $U(x)$ and $V(x)$, inhabiting the region $\Omega$. Moreover, we are assuming that $\Omega$ is fully surrounded by inhospitable areas, because both population densities are subject to homogeneous Dirichlet boundary conditions. Nonlinear diffusion arises mainly in the porous media equation and it was introduced in population dynamics in [16]. We refer to [12] and the references therein for details about the model. For the present model $U$ and $V$ can interact in three different ways indicated by the sign of the last terms in the equations: if both of them are negative then $U$ and $V$ compete; if they are positive then $U$ and $V$ co-operate; and if, for example, the sign is positive in the first equation and negative in the second one then $U$ and $V$ represent the predator and prey populations respectively.

To study (1), we make an appropiate change of variables (see [12]) and obtain

$$
\begin{cases}-\Delta w^{m}=w(\lambda-w \pm b z) & \text { in } \Omega, \\ -\Delta z^{n}=z(\mu-z \pm c w) & \text { in } \Omega, \\ w=z=0 & \text { on } \partial \Omega\end{cases}
$$

where $\lambda, \mu \in \mathbb{R}$ and $b, c>0$.

When $m=n=1,(2)$ is the classical Lotka-Volterra model which has been widely studied in the last years: see [4], [6], [8], [9], [15], [23], [26], [28] in competition, [4], [9], [10], [21], [25] in predator-prey and [11], [19], [22], [24], [27] in symbiosis, for instance.

When $m, n>1$ there exists an important change in the behaviour of the solutions of (2). More precisely, it is possible that one species (or both) is nonnegative but not strictly positive, i.e. there exist some subsets of $\Omega$ with strictly positive measures where the species does not survive. We call them "dead cores" (see [14]). For that, we distinguish two types of nontrivial nonnegative solutions of (2); those with both components positive, the coexistence states, and those where at least one component could have a dead core, the semi-coexistence states.

We now describe the distribution and the contents of this work. In Section 2 we study the single boundary value problem

$$
\left\{\begin{aligned}
-\Delta w^{m} & =w(a(x)-d w) & & \text { in } \Omega, \\
w & =0 & & \text { on } \partial \Omega,
\end{aligned}\right.
$$

which appears when one of the species is zero and where $a \in C^{\alpha}(\bar{\Omega}), \alpha \in(0,1), m \geq 1$ and $d \geq 0$. If $m=1$ it is known that (3) admits a unique positive solution if and only if $\sigma_{1}^{\Omega}(-a)<0$, where $\sigma_{1}^{\Omega}(q)$ stands for the principal eigenvalue of the problem

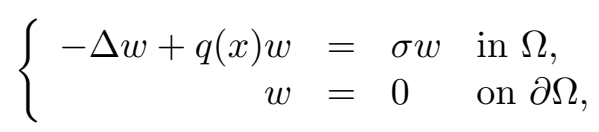


with $q \in L^{\infty}(\Omega)$. The particular case $m>1$ and $d=0$ has been studied in [1], [2], [3] and [17]. Thus, we focus on the case $m>1$ and $d>0$ which has been previously analyzed in [17] and [30]. To state our main results, we need the following notation. For any $a \in C^{\alpha}(\bar{\Omega})$ we denote

$$
a_{L}:=\min _{x \in \bar{\Omega}} a(x) \quad a_{M}:=\max _{x \in \bar{\Omega}} a(x) .
$$

In Theorem 1 we obtain some results concerning existence and uniqueness of nonnegative solutions of (3) which can be summarized as follows:

- If $a_{L} \geq 0$ and $1<m \leq 2$ then there exists a unique positive solution of (3).

- If $a_{M}>0$ then there exists at least a nonnegative solution of (3).

- If $a_{M} \leq 0$ then (3) does not admit a nonnegative solution.

Particular attention is paid to the existence of a dead core for solutions of (3). We show:

- If either $a_{L}>0$ or $a_{L}=0$ and $1<m \leq 2$, any solution of (3) is positive and so it has not a dead core.

- If $a_{L}=0, m>2$ and $A_{0}:=\operatorname{int}\{x \in \Omega: a(x)=0\} \neq \emptyset$, there exists a positive constant $C=C(N, d, \varepsilon)$ such that if $a_{M}<C$ then any nonnegative solution of (3) has a dead core, where $\varepsilon>0$ is the radius of a ball contained in $A_{0}$.

When $a$ changes sign, we write $a_{t}(x):=a^{+}(x)-t a^{-}(x)$ where $a^{ \pm}(x):=\max \{0, \pm a(x)\}$ and for any $t>0$ and $R>0$ we consider the sets

$$
N(R, t):=\left\{x \in \Omega: a^{-}(x) \geq \frac{R}{t}\right\}, \quad M(R, t):=\left\{x \in N(R, t): \operatorname{dist}(x, \partial N(R, t) \backslash \partial \Omega) \geq C_{R}\right\},
$$

with

$$
C_{R}:=\sqrt{\frac{N}{2}} \int_{0}^{\left(\frac{a_{M}^{+}}{d}\right)^{m}} \frac{d s}{\left[\int_{0}^{s}\left(R \nu^{1 / m}+d \nu^{2 / m}\right) d \nu\right]^{1 / 2}} .
$$

With this notation, we obtain that if $M(R, t) \neq \emptyset$ for some $t>0$ and $R>0$ then any nonnegative solution of (3) has a dead core. Moreover, we consider $t$ as parameter and we obtain information about the qualitative behaviour of the nonnegative solutions of (3). Our results in this direction can be stated in the following general terms:

- There exists $t_{0}>0$ such that if $t \geq t_{0}$ then any nonnegative solution of (3) has a dead core.

- If $1<m \leq 2$ there exists $t_{1}>0$ such that if $0<t \leq t_{1}$ then any nonnegative solution of (3) is positive.

- If $m>2$ there exist $d_{0}>0$ and $t_{0}\left(d_{0}\right)>0$ such that if $0<t \leq t_{0}\left(d_{0}\right)$ then any nonnegative solution of (3) has a dead core. 
These results improve and extend those in [30] (See Remark 5 and Section 2).

In Section 3 we analyze the system (2) in the three classical interactions: predator-prey, competition and symbiosis. The prey-predator case has been investigated in [7], [18], [20], [29], [31]; competition one in [7], [29]; and lastly, the symbiosis one in [7]. We consider $\lambda$ and $\mu$ as principal parameters and we study the set of values of $(\lambda, \mu)$ for which (2) admits a semi-coexistence or coexistence state. On the other hand, we obtain a priori bounds for nonnegative solutions of (2) which are the key to get the non-existence regions of nonnegative solutions in the $(\lambda, \mu)$-plane. We should notice that our results improve in some sense those in the above mentioned works (see Remarks 8, 9 and 11).

Now, we are interested in the existence of a dead core for any nonnegative solution of (2). This has been previously studied in the cases of prey-predator and competition in [18] and [29]. To get results concerning this subject, we apply the results of Section 2 and obtain for the prey-predator case:

- For fixed $\lambda>0$, there exists $\mu_{0}(\lambda)>0$ such that if $0<\mu<\mu_{0}(\lambda)$ any nonnegative solution of (2) has a dead core.

- For fixed $\mu>0$, there exist $\lambda_{0}(\mu)<0$ and $\varepsilon>0$ such that if $\lambda \leq \lambda_{0}(\mu)$ there is no nonnegative solution of (2) and if $\lambda \in\left(\lambda_{0}(\mu), \lambda_{0}(\mu)+\varepsilon\right)$ any nonnegative solution of (2) has a dead core.

- For fixed $\lambda, \mu, b>0$, there exists $c_{0}>0$ such that if $c>c_{0}$ any nonnegative solution of (2) has a dead core.

In the case of competition, we have:

- For fixed $\lambda>0$ (resp. $\mu>0$ ), there exists $\mu_{0}(\lambda)>0$ (resp. $\lambda_{0}(\mu)>0$ ) such that if $0<\mu<\mu_{0}(\lambda)$ (resp. $0<\lambda<\lambda_{0}(\mu)$ ) any nonnegative solution of (2) has a dead core.

- For fixed $\lambda, \mu>0$ and $c>0$ (resp. $b>0$ ), there exists $b_{0}>0$ (resp. $c_{0}>0$ ) such that if $b>b_{0}$ (resp. $c>c_{0}$ ) any nonnegative solution of (2) has a dead core.

Moreover, in this Section we will give a biological interpretation to these results.

\section{The logistic equation with nonlinear diffusion}

In this paper we use the following notation: $\Omega$ is a bounded domain in $\mathbb{R}^{N}$ with a smooth enough boundary $\partial \Omega$. For fixed $\alpha>0$, we consider the spaces $\mathcal{U}:=\left\{w \in C^{2, \alpha}(\bar{\Omega}): w=0\right.$ on $\left.\partial \Omega\right\}$ and $\mathcal{V}:=C^{\alpha}(\bar{\Omega})$ ordered by their cones of nonnegative functions $P_{\mathcal{U}}:=\{w \in \mathcal{U}: w \geq 0\}$ and $P_{\mathcal{V}}:=\{w \in \mathcal{V}: w \geq 0\}$. We will write $f \geq g$ if $f-g \in P, f>g$ if $f-g \in P-\{0\}$ and $f \gg g$ if $f-g \in \dot{P}$, where $\dot{P}$ denotes the interior of $P$. Moreover, for any $f \in \mathcal{V}$ we denote

$$
f_{M}:=\max _{x \in \bar{\Omega}} f(x) \quad f_{L}:=\min _{x \in \bar{\Omega}} f(x)
$$


Let $a \in \mathcal{V}, m>1$ and $d>0$, we consider the logistic equation with nonlinear diffusion, namely

$$
\left\{\begin{aligned}
-\Delta w^{m} & =a(x) w-d w^{2} & & \text { in } \Omega, \\
w & =0 & & \text { on } \partial \Omega .
\end{aligned}\right.
$$

Given $q \in L^{\infty}(\Omega), \sigma_{1}^{\Omega}(q)$ stands for the first eigenvalue of

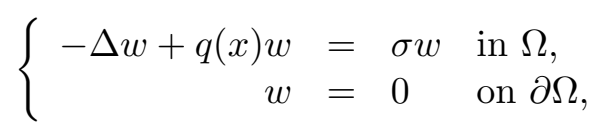

whose corresponding associated eigenfunction $\varphi_{1}^{\Omega}[q]$ can be chosen such that $\varphi_{1}^{\Omega}[q] \gg 0$ and $\left\|\varphi_{1}^{\Omega}[q]\right\|_{\infty}=1$. Moreover, due to the strong maximun principle, we have

$$
\frac{\partial \varphi_{1}^{\Omega}[q]}{\partial n}<0
$$

where $n$ is the outward unit normal at $\partial \Omega$. Finally, we write $\sigma_{1}^{\Omega}:=\sigma_{1}^{\Omega}(0)$ and $\varphi_{1}^{\Omega}:=\varphi_{1}^{\Omega}[0]$. To study (4), we perform the change of variables $w^{m}=u$ and we obtain

$$
\left\{\begin{aligned}
-\Delta u & =a(x) u^{1 / m}-d u^{2 / m} & & \text { in } \Omega, \\
u & =0 & & \text { on } \partial \Omega .
\end{aligned}\right.
$$

For the existence and uniqueness of nonnegative solutions of (7), the main result is the following one:

Theorem 1 Let $a \in \mathcal{V}, d>0$ and $m>1$. The following assertions are true:

1. If $a \gg 0$, then there exists a solution $u \in \mathcal{U}$ of (7) with $u \gg 0$. Moreover, if $m \leq 2$ the solution is unique.

2. If $a>0$ and $m \leq 2$, then there exists a unique solution $u \in \mathcal{U}$ of (7) with $u \gg 0$.

3. If there exists $x_{0} \in \Omega$ such that $a\left(x_{0}\right)>0$, then there exists at least a solution $u \in \mathcal{U}$ of (7) with $u>0$.

Remark 1 If $a \leq 0$ then $u=0$ is the unique nonnegative solution of (7) owing to the maximum principle. On the other hand, observe that the function a can change of sign in case 3.

Proof. We will apply the sub-supersolution method. By the regularity of $a$ there exist $x_{0} \in \Omega$ and $r>0$ such that

$$
a(x) \geq a_{0}>0, \quad \text { for } x \in B\left(x_{0}, r\right),
$$

where $B\left(x_{0}, r\right)$ is the ball of radius $r>0$ centered at $x_{0}$. Now, we define the function

$$
\phi(x):= \begin{cases}\varphi_{1}^{\Omega^{\prime}}(x) & \text { if } x \in \Omega^{\prime}, \\ 0 & \text { if } x \in \Omega \backslash \Omega^{\prime},\end{cases}
$$


with $\Omega^{\prime}=\Omega$ in the case 1 and $\Omega^{\prime}=B\left(x_{0}, r\right)$ in the other cases. We will show that $\underline{u}=\rho \phi$ is a subsolution of (7) with $\rho$ a positive constant. Indeed, we can take $\rho>0$ such that

$$
-\Delta\left(\rho \varphi_{1}^{\Omega^{\prime}}\right) \leq a(x)\left(\rho \varphi_{1}^{\Omega^{\prime}}\right)^{1 / m}-d\left(\rho \varphi_{1}^{\Omega^{\prime}}\right)^{2 / m} \text { in } \Omega^{\prime} .
$$

By (6) we can use Lemma I.1 of [3] and conclude that $\underline{u}$ is a subsolution of (7). As a supersolution we pick $\bar{u}=M$, with $M>0$ a sufficiently large constant. Moreover, it is easy to see that

$$
\|u\|_{\infty} \leq M:=\left(\frac{a_{M}}{d}\right)^{m}
$$

for any solution $u$ of (7). So, there exists at least a solution $u \in \mathcal{U}$ such that $\underline{u} \leq u \leq \bar{u}$.

Clearly $u \gg 0$ in the first case. Now, we show that $u \gg 0$ in the case 2 by applying the strong maximum principle. Let $u>0$ be a solution of (7); it suffices to find a constant $K>0$ such that $(-\Delta+K) u>0$, or equivalently

$$
K>d u^{2 / m-1}-a(x) u^{1 / m-1}, \quad \text { with } u \in(0, M],
$$

which follows from hypotheses and so $u \gg 0$.

For the uniqueness we use [5]. We define the map

$$
t \mapsto g(x, t):=\frac{a(x) t^{1 / m}-d t^{2 / m}}{t}=a(x) t^{1 / m-1}-d t^{2 / m-1} .
$$

It is not hard to prove that this application is decreasing in the cases $a \gg 0$ and $m \leq 2$ as well as $a>0$ and $m<2$. It remains to prove the uniqueness in the case $m=2$ and $a>0$. Let $u_{1}$ and $u_{2}$ be two arbitrary solutions of (7). Now, the function $g$ is nonincreasing, so by the Remark 1 of [5], it follows that there exists a constant $C$ such that $u_{1}=C u_{2}$. Hence,

$$
a(x) u_{1}^{1 / 2}-d u_{1}=-\Delta u_{1}=-\Delta\left(C u_{2}\right)=C\left(a(x) u_{2}^{1 / 2}-d u_{2}\right)=C a(x) u_{2}^{1 / 2}-d u_{1}
$$

from which

$$
a(x) C^{1 / 2} u_{2}^{1 / 2}\left(1-C^{1 / 2}\right)=0,
$$

and therefore $C=1$. This completes the proof.

Remark 2 1.- When $a_{L}>0$ there exists a unique positive solution of (7) provided that

$$
m>2 \text { and } \quad \frac{a_{L}}{a_{M}}>\frac{m-2}{m-1} .
$$

Indeed, it can be proved that the function $t \mapsto g(x, t)$ defined in the proof of Theorem 1 is decreasing.

2.- In the particular case $a(x) \equiv \lambda=$ cte it is known, cf. [13], that there exists a unique positive solution of (7) if and only if $\lambda>0$.

3.- When $a \gg 0$ and $m>1$, any solution of $(7)$ is positive in $\Omega$. In this case, it is easy to prove the existence of the constant $K$ satisfying (9). 
¿From the priori bound (8) follows the existence of a nontrivial maximal solution of (7) which we denote by $\theta_{[a, d, m]}$. We will take $\theta_{[a, d, m]}=0$ if $a \leq 0$.

The next result will be very useful to compare positive solutions of different logistic boundary value problems.

Proposition 1 Let $a, b \in \mathcal{V}$.

1. If $\underline{u}$ is a subsolution of (7), then

$$
\underline{u} \leq \theta_{[a, d, m]}
$$

2. If $a \leq b$, then

$$
\theta_{[a, d, m]} \leq \theta_{[b, d, m]} .
$$

Proof. Let $\underline{u}$ be a subsolution of (7). As sufficiently large constants, say $K>0$, are supersolutions, then there exists a solution $u$ of (7) such that $\underline{u} \leq u \leq K$. From the maximality of $\theta_{[a, d, m]}$ it follows that $u \leq \theta_{[a, d, m]}$. For the second part it is enough to prove that $\theta_{[a, d, m]}$ is a subsolution of

$$
\left\{\begin{aligned}
-\Delta w & =b(x) w^{1 / m}-d w^{2 / m} & & \text { in } \Omega, \\
w & =0 & & \text { on } \partial \Omega,
\end{aligned}\right.
$$

and apply the previous result.

\subsection{Existence of a "dead core"}

In this subsection we shall consider the existence of a "dead core" for solutions $u$ of (7), i.e., we will show that the set $\Omega_{0}:=\{x \in \Omega: u(x)=0\}$ has a strictly positive measure under suitable easily checked hypotheses on $a, d, m, \Omega$ and $N$.

Assume that $a>0$ and

$$
A_{0}:=\operatorname{int}\{x \in \Omega: a(x)=0\} \neq \emptyset .
$$

Theorem 1 ensures that if $m \leq 2$ any nonnegative solution of (7) is positive. Our principal result is:

Theorem 2 Let $m>2, a>0$ and $A_{0}=\operatorname{int}\{x \in \Omega: a(x)=0\} \neq \emptyset$. Let $x_{0} \in A_{0}$ and $\varepsilon>0$ be such that $B\left(x_{0}, 2 \varepsilon\right) \subset A_{0}$ and assume that

$$
a_{M}<\frac{d^{\frac{m-1}{m-2}} \varepsilon^{\frac{2}{m-2}}}{\left[q\left(q-1+\frac{N-1}{2}\right)\right]^{\frac{1}{m-2}}},
$$

with $q=2 m /(m-2)$. Then there exists a dead core for any $u>0$ solution of ( 7$)$. Moreover, we have

$$
\Omega_{0}=\{x \in \Omega: u(x)=0\} \supset B\left(x_{0}, \varepsilon\right)
$$


Proof. Let $x_{0} \in A_{0}$ and $\varepsilon>0$ be such that $B\left(x_{0}, 2 \varepsilon\right) \subset A_{0}$. We will build a function which is zero in $B\left(x_{0}, \varepsilon\right) \subset \Omega$ and bigger than the maximal solution of $(7)$. Without loss of generality we can suppose that $x_{0}=0$. We consider the function

$$
\Psi(x):= \begin{cases}0 & \text { if } x \in B(0, \varepsilon), \\ \frac{(|x|-\varepsilon)^{q}}{\varepsilon^{q}} & \text { if } x \in B(0,2 \varepsilon) \backslash B(0, \varepsilon), \\ 1 & \text { if } x \in \Omega \backslash B(0,2 \varepsilon) .\end{cases}
$$

It is clear that $\Psi \in H^{1}(\Omega)$. We will show that the function $u^{0}:=K \Psi$ satisfies

$$
\theta_{[a, d, m]} \leq u^{0} \quad \text { in } \Omega,
$$

with an appropiate constant $K$. Taking

$$
K \geq\left(\frac{a_{M}}{d}\right)^{m}
$$

we have $u^{0} \geq \theta_{[a, d, m]}$ in $\Omega \backslash B(0,2 \varepsilon)$ from (8). We will prove now that $\left(\theta_{[a, d, m]}-u^{0}\right)^{+}=0$ in $B(0,2 \varepsilon)$. It is sufficient to prove that

$$
\int_{B(0,2 \varepsilon)}\left|\nabla\left(\theta_{[a, d, m]}-u^{0}\right)^{+}\right|^{2} \leq 0
$$

because $\left(\theta_{[a, d, m]}-u^{0}\right)^{+}=0$ on $\partial B(0,2 \varepsilon)$. Let $\phi \in H_{0}^{1}(B(0,2 \varepsilon)), \phi \geq 0$. Then

$$
\begin{gathered}
\int_{B(0,2 \varepsilon)} \nabla\left(\theta_{[a, d, m]}-u^{0}\right) \cdot \nabla \phi=\int_{B(0,2 \varepsilon)} \nabla \theta_{[a, d, m]} \cdot \nabla \phi-K \int_{B(0,2 \varepsilon) \backslash B(0, \varepsilon)} \nabla \Psi \cdot \nabla \phi= \\
\int_{B(0,2 \varepsilon)} \theta_{[a, d, m]}^{1 / m}\left(a(x)-d \theta_{[a, d, m]}^{1 / m}\right) \phi+K \int_{B(0,2 \varepsilon) \backslash B(0, \varepsilon)} \Delta \Psi \cdot \phi-K \int_{\partial B(0, \varepsilon)} \frac{\partial \Psi}{\partial n} \phi .
\end{gathered}
$$

Now using that $\partial \Psi / \partial n=0$ on $\partial B(0, \varepsilon)$ and $a(x)=0$ in $B(0,2 \varepsilon)$, we find that

$$
\begin{gathered}
\int_{B(0,2 \varepsilon)} \nabla\left(\theta_{[a, d, m]}-u^{0}\right) \cdot \nabla \phi \leq-\int_{B(0,2 \varepsilon)} d \theta_{[a, d, m]}^{2 / m} \phi+K \int_{B(0,2 \varepsilon) \backslash B(0, \varepsilon)} \Delta \Psi \cdot \phi= \\
=-\int_{B(0, \varepsilon)} d \theta_{[a, d, m]}^{2 / m} \phi+\int_{B(0,2 \varepsilon) \backslash B(0, \varepsilon)}\left(-d \theta_{[a, d, m]}^{2 / m}+K\left(\frac{q}{\varepsilon^{q}}(|x|-\varepsilon)^{q-2}\left(q-1+\frac{N-1}{|x|}(|x|-\varepsilon)\right)\right)\right) \phi .
\end{gathered}
$$

Taking $\phi=\left(\theta_{[a, d, m]}-u^{0}\right)^{+}$, we obtain

$$
\begin{gathered}
\int_{B(0,2 \varepsilon)}\left|\nabla\left(\theta_{[a, d, m]}-u^{0}\right)^{+}\right|^{2} \leq \\
\leq \int_{B(0,2 \varepsilon) \backslash B(0, \varepsilon)}\left(-d \theta_{[a, d, m]}^{2 / m}+K\left(\frac{q}{\varepsilon^{q}}(|x|-\varepsilon)^{q-2}\left(q-1+\frac{N-1}{|x|}(|x|-\varepsilon)\right)\right)\right)\left(\theta_{[a, d, m]}-u^{0}\right)^{+} .
\end{gathered}
$$

Using (13) and noting that $q-2=2 q / m$, to prove (12) it is sufficient to show that

$$
K^{1-2 / m} \frac{q}{\varepsilon^{2}}\left(q-1+\frac{N-1}{|x|}(|x|-\varepsilon)\right)-d \leq 0 \quad x \in B(0,2 \varepsilon) \backslash B(0, \varepsilon) .
$$


We denote

$$
K_{1}:=\frac{K^{1-2 / m}}{\varepsilon^{2}} q(q-1)-d \quad \text { and } \quad K_{2}:=\frac{K^{1-2 / m}}{\varepsilon^{2}} q(N-1) .
$$

We must prove that

$$
K_{1}+K_{2} \leq \varepsilon \frac{K_{2}}{|x|} \quad \text { for } \varepsilon<|x|<2 \varepsilon .
$$

Assume that $N \geq 2$. If $K_{1}+K_{2} \leq 0$, (14) is trivial because $K_{2}>0$. If $K_{1}+K_{2}>0$, (14) is equivalent to

$$
|x| \leq \varepsilon \frac{K_{2}}{K_{1}+K_{2}}
$$

This inequality is true in $B(0,2 \varepsilon) \backslash B(0, \varepsilon)$ if

$$
2 K_{1}+K_{2} \leq 0
$$

If $N=1$ then $K_{2}=0$, and so (15) is equivalent to be $K_{1} \leq 0$. It is not hard to prove from (10) the existence of a constant $K$ satisfying (11) and (15). This finishes the proof.

Remark 3 We remark that the constant

$$
C:=C(d, \varepsilon, m, N)=\frac{d^{\frac{m-1}{m-2}} \varepsilon^{\frac{2}{m-2}}}{\left[q\left(q-1+\frac{N-1}{2}\right)\right]^{\frac{1}{m-2}}}
$$

satisfies $C \uparrow+\infty$ as $d \uparrow+\infty$ or $\varepsilon \uparrow+\infty$. Then, if $a>0$ is given, $\Omega_{0}$ exists if $d$ is large enough. On the other hand, if $d$ is given, $\Omega_{0}$ exists if $a_{M}$ is sufficiently small or if $A_{0}$ is large.

Now we consider the case where $a$ changes sign. Let $t \in \mathbb{R}^{+}$and we define

$$
a_{t}(x):=a^{+}(x)-t a^{-}(x)
$$

where $a^{ \pm}(x):=\max \{0, \pm a(x)\}$ and we suppose that $a^{+} \not \equiv 0$. The aim is now to study the existence of a dead core of solutions of

$$
\left\{\begin{aligned}
-\Delta u & =a_{t}(x) u^{1 / m}-d u^{2 / m} & & \text { in } \Omega, \\
u & =0 & & \text { on } \partial \Omega,
\end{aligned}\right.
$$

with $t$ as a parameter. To prove the main result we need the following Lemma motivated by Lemma 7 in [30].

Lemma 1 Let $R>0$,

$$
R_{0}:=\sqrt{\frac{N}{2}} \int_{0}^{+\infty} \frac{d s}{\left[\int_{0}^{s}\left(R \nu^{1 / m}+d \nu^{2 / m}\right) d \nu\right]^{1 / 2}}
$$


and $h_{R}:\left[0, R_{0}\right) \mapsto \overline{\mathbb{R}}_{+}$, be implicitly defined by

$$
\int_{0}^{h_{R}(r)} \frac{d s}{\left[\int_{0}^{s}\left(R \nu^{1 / m}+d \nu^{2 / m}\right) d \nu\right]^{1 / 2}}=\sqrt{\frac{2}{N}} r .
$$

Then $h_{R} \in C^{2}\left(0, R_{0}\right), h_{R}(0)=h_{R}^{\prime}(0)=0$ and for any $x, x_{0} \in \mathbb{R}^{N}$

$$
-\Delta h_{R}\left(\left|x-x_{0}\right|\right)+d h_{R}^{2 / m}\left(\left|x-x_{0}\right|\right)+R h_{R}^{1 / m}\left(\left|x-x_{0}\right|\right) \geq 0 .
$$

Proof. Firstly, observe that $R_{0}=+\infty$ if $m \geq 2$ and $R_{0}<+\infty$ if $m<2$. It is not difficult to prove that for any $r_{0}>0$ there exists a unique value of $h_{R}\left(r_{0}\right)$ satisfying (17). Moreover, $h_{R}(0)=0$ and the first claim follows from the implicit function theorem. On the other hand, from (17) it is easy to show that

$$
h_{R}^{\prime}(r)=\sqrt{\frac{2}{N}}\left[\int_{0}^{h_{R}(r)}\left(R s^{1 / m}+d s^{2 / m}\right) d s\right]^{1 / 2} \quad h_{R}^{\prime \prime}(r)=\frac{1}{N}\left(R h_{R}^{1 / m}(r)+d h_{R}^{2 / m}(r)\right) .
$$

Hence,

$$
\begin{gathered}
-\Delta h_{R}\left(\left|x-x_{0}\right|\right)+d h_{R}^{2 / m}\left(\left|x-x_{0}\right|\right)+R h_{R}^{1 / m}\left(\left|x-x_{0}\right|\right)= \\
=-h_{R}^{\prime \prime}(r)-\frac{N-1}{r} h_{R}^{\prime}(r)+d h_{R}^{2 / m}+R h_{R}^{1 / m}(r)=\frac{N-1}{N r} g(r),
\end{gathered}
$$

where

$$
g(r)=\left(R h_{R}^{1 / m}(r)+d h_{R}^{2 / m}(r)\right) r-\sqrt{2 N}\left[\int_{0}^{h_{R}(r)}\left(R s^{1 / m}+d s^{2 / m}\right) d s\right]^{1 / 2} .
$$

It is clear that $g(0)=0$ and

$$
g^{\prime}(r)=\frac{r}{m}\left[R h_{R}(r)^{(1-m) / m}+2 d h_{R}(r)^{(2-m) / m}\right] h_{R}^{\prime}(r),
$$

and so $g \geq 0$. This completes the proof.

We consider the sets

$$
N(R, t):=\left\{x \in \Omega: a^{-}(x) \geq \frac{R}{t}\right\}, \quad M(R, t):=\left\{x \in N(R, t): \operatorname{dist}(x, \partial N(R, t) \backslash \partial \Omega) \geq C_{R}\right\},
$$

where

$$
C_{R}:=\sqrt{\frac{N}{2}} \int_{0}^{\left(\frac{a_{M}^{+}}{d}\right)^{m}} \frac{d s}{\left[\int_{0}^{s}\left(R \nu^{1 / m}+d \nu^{2 / m}\right) d \nu\right]^{1 / 2}} .
$$

The main result is the following one:

Theorem 3 Assume $m>1$ and that for some $R>0$ and $t>0, M(R, t) \neq \emptyset$. Then there exists a dead core for any solution $u$ of (16). Moreover, we have

$$
\Omega_{0}=\{x \in \Omega: u(x)=0\} \supset M(R, t) .
$$


Proof. Let $x_{0} \in M(R, t)$. Then

$$
B\left(x_{0}, C_{R}\right) \subset N(R, t)
$$

We consider the map

$$
w(x):=h_{R}\left(\left|x-x_{0}\right|\right),
$$

which is well-defined in $B\left(x_{0}, C_{R}\right)$ because $C_{R}<R_{0}$. Hence, by Lemma 1 , it follows that

$$
-\Delta\left(w-\theta_{\left[a_{t}, d, m\right]}\right) \geq R\left(\theta_{\left[a_{t}, d, m\right]}^{1 / m}-w^{1 / m}\right)+d\left(\theta_{\left[a_{t}, d, m\right]}^{2 / m}-w^{2 / m}\right) \quad \text { in } B\left(x_{0}, C_{R}\right) .
$$

Now we study the behaviour on $\partial B\left(x_{0}, C_{R}\right)$. If $x \in \partial B\left(x_{0}, C_{R}\right)$, then

$$
w(x)=h_{R}\left(C_{R}\right)=\left(\frac{a_{M}^{+}}{d}\right)^{m} \geq \theta_{\left[a_{t}, d, m\right]}(x) .
$$

The maximum principle implies that $w \geq \theta_{\left[a_{t}, d, m\right]}$ in $B\left(x_{0}, C_{R}\right)$. Since $w\left(x_{0}\right)=0$, then $\theta_{\left[a_{t}, d, m\right]}\left(x_{0}\right)=0$. This concludes the proof.

Remark 4 We could formulate a similar result with $R=0$ assuming that $m>2$.

It seems reasonable that for large $t,(16)$ should have a dead core. The following result shows this fact.

Corollary 1 Let $m>1$ and $d>0$. Then there exists $t_{0}>0$ such that for $t \geq t_{0}$ any solution of (16) has a dead core.

Proof. We take $R(t)=t^{1 / 2}$. Then

$$
N(R(t), t)=\left\{x \in \Omega: a^{-}(x) \geq \frac{1}{t^{1 / 2}}\right\} .
$$

It is easy to show that if $t_{1} \leq t_{2}$ then $N\left(R\left(t_{1}\right), t_{1}\right) \subset N\left(R\left(t_{2}\right), t_{2}\right)$. On the other hand, there exist $\bar{t}>0$ (it is enough to take $\bar{t}>\frac{1}{\left(a^{-}\right)_{M}^{2}}$ ), $x_{0} \in \Omega$ and $r_{0}>0$ such that

$$
B\left(x_{0}, r_{0}\right) \subset N(R(\bar{t}), \bar{t}) \subset N(R(t), t), \quad \text { for any } t \geq \bar{t} .
$$

Since $C_{R(t)} \downarrow 0$ as $t \uparrow \infty$, there exists $\underline{t}>0$ such that if $t \geq \underline{t}$ then

$$
C_{R(t)}<r_{0}
$$

Hence, taking $t_{0}:=\max \{\bar{t}, \underline{t}\}$, for $t \geq t_{0}$ we obtain

$$
\operatorname{dist}\left(x_{0}, \partial N(R(t), t) \backslash \partial \Omega\right) \geq \operatorname{dist}\left(x_{0}, \partial B\left(x_{0}, r_{0}\right) \backslash \partial \Omega\right)=r_{0}>C_{R(t)},
$$

and therefore $M(R(t), t) \neq \emptyset$. The result now follows from Theorem 3 .

The case $t=0$ has been studied in Theorem 2. We now see what happens when $t$ is positive and small. In [30] the authors presented an example in which there is not a dead core when $t$ is positive and small. Their reasoning can be generalized in the spatial dimension as well as the generality of $a_{t}$. The proof of this result is rather similar to the one written in [30] and so we omit it here. 
Corollary 2 Let $1<m \leq 2$. Then there exists $t_{0}>0$ such that for $0<t \leq t_{0}$, any solution of (16) is positive.

The following result says us that the above one is not true in general in the case $m>2$.

Corollary 3 Assume $m>2$. Then there exist $d_{0}>0$ and $t_{0}\left(d_{0}\right)>0$ such that for $0<t \leq t_{0}$, any solution of (16) has a dead core.

Proof. We take $R(t)=t^{2}$. Then $N(R(t), t)=\left\{x \in \Omega: a^{-}(x) \geq t\right\}$. Let $x_{0} \in \Omega$ be such that $a^{-}\left(x_{0}\right)=\left(a^{-}\right)_{M}$ and let $\mathcal{C}$ be the connected component of $\Omega$ such that $x_{0} \in \mathcal{C}$. Let $r_{0}>0$ be such that $B\left(x_{0}, r_{0}\right) \subset \mathcal{C}$ and

$$
0<\underline{a}:=\left(a^{-}\right)_{L} \quad \text { in } B\left(x_{0}, r_{0}\right) .
$$

Since $C_{R(t)}<C_{0}$ and $C_{0} \downarrow 0$ as $d \uparrow \infty$ (see Remark 4), then there exists $d_{0}>0$ such that

$$
C_{R(t)}<r_{0} \quad \text { for } d \geq d_{0}
$$

Let $0<t_{0} \leq \underline{a}$. Then, for $t \leq t_{0}$ we get

$$
B\left(x_{0}, r_{0}\right) \subset N\left(R\left(t_{0}\right), t_{0}\right) \subset N(R(t), t) .
$$

As in Corollary 1 it follows that $M\left(R\left(t_{0}\right), t_{0}\right) \neq \emptyset$. This completes the proof.

Remark 5 1. Note that given $t>0$, if $a_{M} \downarrow 0$ or $d \uparrow+\infty$ then there exists a dead core of the solutions of (16). Indeed, for fixed $t_{0}>0$, let $x_{0} \in \Omega$ be such that $a^{-}\left(x_{0}\right)=\left(a^{-}\right)_{M}>0$. Then there exist $R_{0}>0$ sufficiently small $\left(0<R_{0}<\left(a^{-}\right)_{M}\right)$ and $r_{0}\left(R_{0}\right)>0$ such that

$$
B\left(x_{0}, r_{0}\left(R_{0}\right)\right) \subset N\left(R_{0}, t_{0}\right) .
$$

Moreover, $C_{R} \downarrow 0$ as $a_{M} \downarrow 0$ or $d \uparrow \infty$. So, we can reason as in the preceding corollaries and prove that $M\left(R_{0}, t_{0}\right) \neq \emptyset$. It is sufficent to apply Theorem 3.

2. We would like to point out that Theorem 3 improves the result obtained in [30] where the authors proved the same result with

$$
\widetilde{C_{R}}:=\sqrt{\frac{N}{2 R}} \int_{0}^{\left(\frac{a_{M}^{+}}{d}\right)^{m}} \frac{d s}{\left[\int_{0}^{s} \nu^{1 / m} d \nu\right]^{1 / 2}}
$$

instead of $C_{R}$. It is clear that $\widetilde{C_{R}}>C_{R}$. 


\section{Systems}

As we have mentioned in the Introduction, in this section we will apply the preceding results to the following systems

$$
\begin{cases}-\Delta w^{m}=w(\lambda-w \pm b z) & \text { in } \Omega, \\ -\Delta z^{n}=z(\mu-z \pm c w) & \text { in } \Omega \\ w=z=0 & \text { on } \partial \Omega,\end{cases}
$$

where $b, c>0$ and $\lambda, \mu \in \mathbb{R}$. Performing the change of variables $w^{m}=u$ and $z^{n}=v$, (18) can be rewritten as

$$
\begin{cases}-\Delta u=\lambda u^{1 / m}-u^{2 / m} \pm b u^{1 / m} v^{1 / n} & \text { in } \Omega, \\ -\Delta v=\mu v^{1 / n}-v^{2 / n} \pm c v^{1 / n} u^{1 / m} & \text { in } \Omega, \\ u=v=0 & \text { on } \partial \Omega .\end{cases}
$$

\subsection{Predator-prey}

We consider the following problem

$$
\begin{cases}-\Delta u=\lambda u^{1 / m}-u^{2 / m}+b u^{1 / m} v^{1 / n} & \text { in } \Omega, \\ -\Delta v=\mu v^{1 / n}-v^{2 / n}-c v^{1 / n} u^{1 / m} & \text { in } \Omega, \\ u=v=0 & \text { on } \partial \Omega\end{cases}
$$

where $u$ and $v$ denote the predator and prey populations respectively.

Lemma 2 The condition $\mu>0$ is necessary for the existence of coexistence and semi-coexistence states.

The proof follows from applying the maximum principle to second equation in (20).

Firstly, we focus our attention on the existence of semi-coexistence states. We consider $(\lambda, \mu)$ as parameters and study the set of values of $(\lambda, \mu)$ in $(\lambda, \mu)$-plane where semi-coexistence states exist.

Hereafter we shall denote $\theta_{[a, m]}:=\theta_{[a, 1, m]}$ and

$$
\theta_{\lambda, 0}:=\theta_{[\lambda, m]}^{1 / m} \quad \theta_{\mu, 0}:=\theta_{[\mu, n]}^{1 / n},
$$

and for $r \geq 0$,

$$
\theta_{\lambda, r+1}:=\theta_{\left[\lambda+b \theta_{\mu, r}, m\right]}^{1 / m} \quad \theta_{\mu, r+1}:=\theta_{\left[\mu-c \theta_{\lambda, r}, n\right]}^{1 / n} .
$$

Then, for $r \geq 1$

$$
-\Delta\left(\theta_{\lambda, r}^{m}\right)=\theta_{\lambda, r}\left(\lambda+b \theta_{\mu, r-1}-\theta_{\lambda, r}\right), \quad-\Delta\left(\theta_{\mu, r}^{n}\right)=\theta_{\mu, r}\left(\mu-c \theta_{\lambda, r-1}-\theta_{\mu, r}\right) .
$$

With respect to the existence of semi-coexistence states, our main result is the following one:

Theorem 4 Assume $\mu>0$ and that the set

$$
\Omega_{1}:=\operatorname{int}\left\{x \in \Omega: \lambda+b \theta_{\mu, 2}(x)>0\right\} \neq \emptyset .
$$

Then there exists at least one semi-coexistence state of (20). 
Proof. We use the sub-supersolution method, see [12], i.e, we have to find $\underline{u}, \bar{u}, \underline{v}, \bar{v} \in H^{1}(\Omega) \cap$ $L^{\infty}(\Omega), \underline{u} \leq \bar{u}$ and $\underline{v} \leq \bar{v}$ in $\Omega, \underline{u} \leq 0 \leq \bar{u}$ and $\underline{v} \leq 0 \leq \bar{v}$ on $\partial \Omega$ and such that for any $\varphi \in H_{0}^{1}(\Omega)$, $\varphi \geq 0$ we have

$$
\left\{\begin{array}{l}
\int_{\Omega} \nabla \underline{u} \cdot \nabla \varphi \leq \int_{\Omega}\left(\lambda \underline{u}^{1 / m}-\underline{u}^{2 / m}+b \underline{u}^{1 / m} \underline{v}^{1 / n}\right) \varphi \\
\int_{\Omega} \nabla \bar{u} \cdot \nabla \varphi \geq \int_{\Omega}\left(\lambda \bar{u}^{1 / m}-\bar{u}^{2 / m}+b \bar{u}^{1 / m} \bar{v}^{1 / n}\right) \varphi \\
\int_{\Omega} \nabla \underline{v} \cdot \nabla \varphi \leq \int_{\Omega}\left(\mu \underline{v}^{1 / n}-\underline{v}^{2 / n}-c \underline{v}^{1 / n} \bar{u}^{1 / m}\right) \varphi \\
\int_{\Omega} \nabla \bar{v} \cdot \nabla \varphi \geq \int_{\Omega}\left(\mu \bar{v}^{1 / n}-\bar{v}^{2 / n}-c \bar{v}^{1 / n} \underline{u}^{1 / m}\right) \varphi
\end{array}\right.
$$

Let $B_{1}$ be an open ball such that $B_{1} \subset \Omega_{1}$. We pick

$$
(\underline{u}, \bar{u})=\left(\rho \phi, \theta_{\lambda, 1}^{m}\right), \quad(\underline{v}, \bar{v})=\left(\theta_{\mu, 2}^{n}, \theta_{\mu, 0}^{n}\right)
$$

where $\rho>0$ has still to be specified and

$$
\phi(x):= \begin{cases}\varphi_{1}^{B_{1}}(x) & \text { if } x \in B_{1}, \\ 0 & \text { if } x \in \Omega \backslash B_{1} .\end{cases}
$$

¿From Proposition 1 we have $\underline{v} \leq \bar{v}$ and we can choose $\rho$ such that $\underline{u} \leq \bar{u}$. We must prove only the inequalities in (23). We will show the first one, the other ones follow from (21) directly. Let $\varphi \in H_{0}^{1}(\Omega), \varphi \geq 0$. Then

$$
\begin{gathered}
\int_{\Omega} \nabla \underline{u} \cdot \nabla \varphi=\rho \int_{B_{1}} \nabla \varphi_{1}^{B_{1}} \cdot \nabla \varphi=-\rho \int_{B_{1}} \Delta \varphi_{1}^{B_{1}} \cdot \varphi+\rho \int_{\partial B_{1}} \frac{\partial \varphi_{1}^{B_{1}}}{\partial n} \cdot \varphi \\
<\rho \int_{B_{1}} \sigma_{1}^{B_{1}} \varphi_{1}^{B_{1}} \cdot \varphi \leq \rho^{1 / m} \int_{\Omega}\left(\lambda \phi^{1 / m}-\rho^{1 / m} \phi^{2 / m}+b \phi^{1 / m} \underline{v}^{1 / n}\right) \varphi=\int_{\Omega} \underline{u}^{1 / m}\left(\lambda-\underline{u}^{1 / m}+b \underline{v}^{1 / n}\right) \varphi
\end{gathered}
$$

taking $\rho$ sufficiently small and using (22). Finally, we claim that $\theta_{\mu, 2}>0$. Indeed, since $\mu>0$ it follows from Theorem 1 that $\theta_{\mu, 0} \gg 0$. Moreover, the set

$$
\Omega_{2}:=\operatorname{int}\left\{x \in \Omega: \mu-c \theta_{\lambda, 1}(x)>0\right\} \neq \emptyset,
$$

and so, the claim follows from Theorem 1. 3). This completes the proof.

Remark 6 Observe that, since $\theta_{\mu, 2}>0$, condition (22) is true for any $\lambda$ such that

$$
\lambda>-b\left(\theta_{\mu, 2}\right)_{M}
$$

The following result gives us a priori bounds for the semi-coexistence states.

Proposition 2 Let $(u, v)$ be a semi-coexistence state of (20). Then

$$
\theta_{[\lambda, m]} \leq u \leq(\lambda+b \mu)^{m}, \quad v \leq \theta_{[\mu, n]}
$$


Proof. Let $(u, v)$ be a semi-coexistence state of (20). It can be proved easily that $v$ is a subsolution of (7) with $a(x)=\mu$. The second relation of (24) follows from Proposition 1.

The left inequality of the first relation of (24) follows as $u$ a supersolution of (7) with $a(x)=\lambda$ and the positive solution of this equation is unique.

Now we show the right inequality. We define $\Omega_{1}=\left\{x \in \Omega: u^{1 / m}(x)>\lambda+b \mu\right\}$. Then,

$$
-\Delta u \leq b u^{1 / m}\left(v^{1 / n}-\mu\right) \leq 0 \quad \text { in } \Omega_{1} .
$$

Hence $\left|\Omega_{1}\right|=0$ and the result follows.

As an easy consequence from Proposition 2 we obtain the following result, which provides us of a non-existence region of nonnegative solutions.

Corollary 4 Assume $\lambda+b \mu \leq 0$. Then (20) does not admit a semi-coexistence state.

Proof. ¿From (24) and (8), we have $\lambda+b v^{1 / n} \leq \lambda+b \mu$. Hence,

$$
-\Delta u=\lambda u^{1 / m}-u^{2 / m}+b u^{1 / m} v^{1 / n}=\left(\lambda+b v^{1 / n}\right) u^{1 / m}-u^{2 / m} \leq(\lambda+b \mu) u^{1 / m}-u^{2 / m} .
$$

The result now follows from Remark 1.

Now we are interested in the existence of coexistence states. We proved in [12] the following result that we include here for the sake of completeness.

Theorem 5 Assume $b c<1$ and

$$
\lambda>0 \quad \text { and } \quad \mu>c(\lambda+b \mu) .
$$

Then there exists at least one coexistence state of (20).

¿From Theorems 4 and 5 a natural question is raised: is there a region in the $(\lambda, \mu)$-plane where (20) possesses at least a semi-coexistence state but not a coexistence state, i.e., a region where any nonnegative solution of (20) has a dead core? We present a couple of results about this subject. The first one provides us a region in the $(\lambda, \mu)$-plane where any nonnegative solution of (20) is positive.

Proposition 3 Assume $b c<1$ and that $\lambda$ and $\mu$ satisfy (25). Then any semi-coexistence state of (20) is a coexistence state, i.e., there is not a dead core.

Proof. Let $(u, v)$ be a semi-coexistence state of $(20)$. Then

$$
-\Delta u=u^{1 / m}\left(\lambda+b v^{1 / n}\right)-u^{2 / m}, \quad-\Delta v=v^{1 / n}\left(\mu-c u^{1 / m}\right)-v^{2 / n} .
$$

On the other hand, if $\lambda$ and $\mu$ satisfy (25), we get $\lambda+b v^{1 / n} \gg 0$ and $\mu-c u^{1 / m} \gg 0$. Hence, using Remark 2. 3) the result follows.

that The following result gives us conditions in terms of the coefficients in the model setting which ensure that any nonnegative solution of (20) has a dead core. 
Theorem 6 The following assertions are true:

1. Assume $\lambda>0$. Then there exists $\rho(\lambda)>0$ such that if $0<\mu<\rho(\lambda)$ any nonnegative solution of (20) has a dead core.

2. Assume $\mu>0$. Then there exists $\lambda_{0}(\mu)<0$ and $\varepsilon>0$ such that if $\lambda \in\left(\lambda_{0}(\mu), \lambda_{0}(\mu)+\varepsilon\right)$ any nonnegative solution of (20) has a dead core.

3. Assume $\mu>0$ and $\lambda>0$. Then there exists $c_{0}>0$ such that if $c>c_{0}$ any nonnegative solution of (20) has a dead core.

Proof. Using Propositions 1 and 2 it is easy to prove that for any nonnegative solution $(u, v)$ of (20) we have

$$
u \leq \theta_{\left[\lambda+b \theta_{[\mu, n]}^{1 / n}, m\right]} \quad v \leq \theta_{\left[\mu-c \theta_{[\lambda, m]}^{1 / m}, n\right]},
$$

and so,

$$
\begin{aligned}
& \Omega_{u, 0}:=\{x \in \Omega: u(x)=0\} \supset\left\{x \in \Omega: \theta_{\left[\lambda+b \theta_{[\mu, n]}^{1 / n}, m\right]}(x)=0\right\}, \\
& \Omega_{v, 0}:=\{x \in \Omega: v(x)=0\} \supset\left\{x \in \Omega: \theta_{\left[\mu-c \theta_{[\lambda, m]}^{1 / m}, n\right]}(x)=0\right\} .
\end{aligned}
$$

To prove the first part we will use Theorem 3 and Remark 5. In this case

$$
a(x)=\mu-c \theta_{[\lambda, m]}^{1 / m}(x) .
$$

Hence $a_{M}=\mu$ and $A^{-}=\left\{x \in \Omega: \mu-c \theta_{[\lambda, m]}^{1 / m}(x)<0\right\}$. Let $x_{0} \in \Omega$ and $R>0$ be such that $\theta_{[\lambda, m]}^{1 / m}\left(x_{0}\right)=\left(\theta_{[\lambda, m]}^{1 / m}\right)_{M}$ and $c\left(\theta_{[\lambda, m]}^{1 / m}\right)_{M}-R>0$. We define

$$
B(x):=c \theta_{[\lambda, m]}^{1 / m}(x)-R,
$$

and so,

$$
N(R, 1):=N(\mu)=\{x \in \Omega: \mu \leq B(x)\} .
$$

It is easily seen that if $\mu_{1} \leq \mu_{2}$ then

$$
N\left(\mu_{2}\right) \subset N\left(\mu_{1}\right) .
$$

Let $\delta>0$ be such that $0<\delta<c\left(\theta_{[\lambda, m]}^{1 / m}\right)_{M}-R$ and define

$$
\mu_{0}:=c\left(\theta_{[\lambda, m]}^{1 / m}\right)_{M}-R-\delta
$$

With these choices we can see that $x_{0} \in N\left(\mu_{0}\right)$ and from the continuity of $B(x)$ there exists $r_{\mu_{0}}>0$ such that $B\left(x_{0}, r_{\mu_{0}}\right) \subset N\left(\mu_{0}\right)$. Moreover, from (27) we get

$$
B\left(x_{0}, r_{\mu_{0}}\right) \subset N\left(\mu_{0}\right) \subset N(\mu) \text { for } \mu \in\left[0, \mu_{0}\right] .
$$

On the other hand, if $\mu \downarrow 0$ then $a_{M}=\mu \downarrow 0$, and so $C_{R} \downarrow 0$. Thus, there exists $\mu_{1}>0$ such that if $0<\mu<\mu_{1}$ then $C_{R}<r_{\mu_{0}}$. Finally, we take $\rho:=\min \left\{\mu_{0}, \mu_{1}\right\}$. Thus, for $\mu \in(0, \rho)$

$$
C_{R}<r_{\mu_{0}} \text { and } B\left(x_{0}, r_{\mu_{0}}\right) \subset N(\mu)
$$


and so,

$$
\operatorname{dist}\left(x_{0}, \partial N(\mu) \backslash \partial \Omega\right) \geq \operatorname{dist}\left(x_{0}, \partial B\left(x_{0}, r_{\mu_{0}}\right) \backslash \partial \Omega\right)=r_{\mu_{0}}>C_{R} .
$$

Therefore $M(R, 1) \neq \emptyset$, and so the first part follows from Theorem 3 .

In order to prove the second part let

$$
a(x)=\lambda+b \theta_{[\mu, n]}^{1 / n}(x)
$$

and so,

$$
a_{M}=\lambda+b\left(\theta_{[\mu, n]}^{1 / n}\right)_{M}
$$

We take

$$
\lambda_{0}=-b\left(\theta_{[\mu, n]}^{1 / n}\right)_{M}
$$

It is clear that if $\lambda>\lambda_{0}$ then $a_{M}>0$. Let $R$ and $\delta$ be such that $0<R<\delta<-\lambda_{0}$. Thus, for $\lambda \in\left(\lambda_{0},-\delta\right]$ the set

$$
N(R, 1)=\left\{x \in \Omega: \lambda \leq-R-b \theta_{[\mu, n]}^{1 / n}(x)\right\} \neq \emptyset .
$$

Observe that if $\lambda \downarrow \lambda_{0}^{+}$then $a_{M} \downarrow 0$, and so $C_{R} \downarrow 0$. Now, reasoning as in the above paragraph, the result follows.

To prove the last part we fix $\lambda, \mu>0$. We take $R=c^{1 / 2}$ and denote by $N(R, 1):=N(c)$. Thus,

$$
N(c)=\left\{x \in \Omega: \frac{\mu+c^{1 / 2}}{c} \leq \theta_{[\lambda, m]}^{1 / m}(x)\right\} .
$$

Observe that the function

$$
f(c):=\frac{\mu+c^{1 / 2}}{c}
$$

is decreasing and tends to 0 as $c \uparrow \infty$. Moreover, if $c_{1} \leq c_{2}$ then $N\left(c_{1}\right) \subset N\left(c_{2}\right)$. Hence, for $c$ sufficiently large we get $N(c) \neq \emptyset$. Finally, observe that $C_{R(c)} \downarrow 0$ as $c \uparrow \infty$. We can complete the proof by using an argument similar to that used previously.

Remark 7 We can give a biological interpretation of the above result. In the part 1 we fix the predator's growth rate. Then if the prey's growth rate is small, the prey do not live in all the space $\Omega$, i.e., there exist some subregions of $\Omega$ where the prey is driven to extinction by the predator. On the other hand, in part 3 if the rate at which the prey is consumed by the predator (the parameter $c$ in the setting of the system (20)) is sufficiently large, then there exist some areas where the prey do not exist.

In part 2, from (26) observe that if $\lambda \leq-b\left(\theta_{[\mu, n]}^{1 / n}\right)_{M}:=\lambda_{0}$ then there are no semi-coexistence states of (20). Theorem 6 ensures that (20) does not admit a coexistence state if $\lambda \in\left(\lambda_{0}, \lambda_{0}+\varepsilon\right)$.

Remark 8 Our results are improvement on previous results papers. Indeed, in [20] the authors obtained under condition (25) the existence of a coexistence state of (20) but only in the particular case $1<m=n<2$. In [7] the authors showed the existence of a semi-coexistence state when $\lambda$ and $\mu$ satisfy (25). It is clear that condition (22) is weaker than (25). 


\subsection{Competition}

Consider the following model whose solutions represent the steady states of dynamical models of competing populations

$$
\begin{cases}-\Delta u=\lambda u^{1 / m}-u^{2 / m}-b u^{1 / m} v^{1 / n} & \text { in } \Omega, \\ -\Delta v=\mu v^{1 / n}-v^{2 / n}-c v^{1 / n} u^{1 / m} & \text { in } \Omega, \\ u=v=0 & \text { on } \partial \Omega .\end{cases}
$$

Concerning the existence of semi-coexistence states, we have the following result:

Theorem 7 (28) possesses a semi-coexistence state if and only if $\lambda>0$ and $\mu>0$.

Proof. Let $(u, v)$ be a semi-coexistence state of (28). Then the maximum principle ensures that $\lambda$ and $\mu$ have to be positive. Assume now $\lambda, \mu>0$. It is clear that

$$
B_{1}:=\left\{x \in \Omega: \lambda-b \theta_{[\mu, n]}^{1 / n}(x)>0\right\} \neq \emptyset, \quad B_{2}:=\left\{x \in \Omega: \mu-c \theta_{[\lambda, m]}^{1 / m}(x)>0\right\} \neq \emptyset .
$$

We have to find a couple $(\underline{u}, \bar{u})-(\underline{v}, \bar{v})$ of sub-supersolutions of $(28)$, see [12], i.e. $\underline{u}, \bar{u}, \underline{v}, \bar{v} \in$ $H^{2}(\Omega) \cap L^{\infty}(\Omega), \underline{u} \leq \bar{u}$ and $\underline{v} \leq \bar{v}$ in $\Omega$ and $\underline{u} \leq 0 \leq \bar{u}$ and $\underline{v} \leq 0 \leq \bar{v}$ on $\partial \Omega$ and such that

$$
\begin{cases}-\Delta \underline{u} \leq \lambda \underline{u}^{1 / m}-\underline{u}^{2 / m}-b \underline{u}^{1 / m} \bar{v}^{1 / n} & \text { in } \Omega, \\ -\Delta \bar{u} \geq \lambda \bar{u}^{1 / m}-\bar{u}^{2 / m}-b \bar{u}^{1 / m} \underline{v}^{1 / n} & \text { in } \Omega, \\ -\Delta \underline{v} \leq \mu \underline{v}^{1 / n}-\underline{v}^{2 / n}-c \underline{v}^{1 / n} \bar{u}^{1 / m} & \text { in } \Omega, \\ -\Delta \bar{v} \geq \mu \bar{v}^{1 / n}-\bar{v}^{2 / n}-c \bar{v}^{1 / n} \underline{u}^{1 / m} & \text { in } \Omega .\end{cases}
$$

We pick

$$
(\underline{u}, \bar{u})=\left(\theta_{\left[\lambda-b \theta_{[\mu, n]}^{1 / n}, m\right]}, \theta_{[\lambda, m]}\right) \quad(\underline{v}, \bar{v})=\left(\theta_{\left[\mu-c \theta_{[\lambda, m]}^{1 / m}, n\right]}, \theta_{[\mu, n]}\right) .
$$

Using (29) and Theorem 1, it can be shown that $\underline{u}>0, \underline{v}>0$. Moreover, from Proposition 1, $\underline{u} \leq \bar{u}$ and $\underline{v} \leq \bar{v}$ in $\Omega$. It is not hard to prove that this couple satisfies the inequalities in (30) and this completes the proof.

The proofs of the following results are similar to the predator-prey ones, so we omit them.

Proposition 4 Let $(u, v)$ be a semi-coexistence state of (28). Then

$$
u \leq \theta_{[\lambda, m]} \quad v \leq \theta_{[\mu, n]} .
$$

With respect to the existence of coexistence states we have the following result whose proof can be found in [12].

Theorem 8 Assume $b c<1$ and

$$
\lambda>b \mu \text { and } \quad \mu>c \lambda .
$$

Then there exists at least one coexistence state of (28). 
Finally, concerning conditions on $\lambda$ and $\mu$ under which any coexistence state has not a dead core, we obtain:

Proposition 5 Assume $b c<1$ and that $\lambda$ and $\mu$ satisfy (32). Then any nonnegative solution of (28) has not a dead core.

The following result gives us conditions which ensure that any nonnegative solution of (28) has a dead core.

Theorem 9 The following assertions are true:

1. Assume $\lambda>0$. Then there exists $\mu(\lambda)>0$ such that if $0<\mu<\mu(\lambda)$ any nonnegative solution of (28) has a dead core.

2. Assume $\mu>0$. Then there exists $\lambda(\mu)>0$ such that if $0<\lambda<\lambda(\mu)$ any nonnegative solution of (28) has a dead core.

3. Assume $\lambda, \mu>0$ and $b>0$. Then there exists $c_{0}>0$ such that if $c>c_{0}$ any nonnegative solution of (28) has a dead core.

4. Assume $\lambda, \mu>0$ and $c>0$. Then there exists $b_{0}>0$ such that if $b>b_{0}$ any nonnegative solution of (28) has a dead core.

Proof. We will prove the first and third parts. The other ones follow similarly. We claim that for any nonnegative solution $(u, v)$ of $(28)$, we have

$$
u \leq \theta_{\left[\lambda-b \theta_{\left[\mu-c \theta_{[\lambda, m]}^{1 / n}, n\right]}^{1 / m}, m\right]}, \quad v \leq \theta_{\left[\mu-c \theta_{\left[\lambda-b \theta_{[\mu, n]}^{1 / n}, m\right]}^{1 / m}, n\right]} .
$$

Indeed, using Proposition 1 it is sufficient to show that $u$ is subsolution of

$$
\left\{\begin{aligned}
-\Delta w & =\left(\lambda-b \theta_{\left[\mu-c \theta_{[\lambda, m]}^{1 / m}, n\right]}^{1 / n}\right) w^{1 / m}-w^{2 / m} & & \text { in } \Omega, \\
w & =0 & & \text { on } \partial \Omega,
\end{aligned}\right.
$$

which is true if $\theta_{\left[\mu-c \theta_{[\lambda, m]}^{1 / m}, n\right]} \leq v$. ¿From Propositions 4 and 1 , it follows that

$$
v=\theta_{\left[\mu-c u^{1 / m}, n\right]} \geq \theta_{\left[\mu-c \theta_{[\lambda, m]}^{1 / m}, n\right]} .
$$

This proves the first relation of (33). The second one follows analogously. So, from (33) we have

$$
\begin{aligned}
& \Omega_{u, 0}=\{x \in \Omega: u(x)=0\} \supset\left\{x \in \Omega: \theta_{\left[\lambda-b \theta_{\left[\mu-c \theta_{[\lambda, m]}^{1 / n}, n\right]}^{1 / m}, m\right]}(x)=0\right\}, \\
& \Omega_{v, 0}=\{x \in \Omega: v(x)=0\} \supset\left\{x \in \Omega: \theta_{\left[\mu-c \theta_{\left[\lambda-b \theta_{[\mu, n]}^{1 / n}, m\right]}^{1 / m}, n\right]}(x)=0\right\} .
\end{aligned}
$$

We now prove the first part. For fixed $\lambda>0$, we consider

$$
a(x)=\mu-c \theta_{\left[\lambda-b \theta_{[\mu, n]}^{1 / n}, m\right]}^{1 / m}(x),
$$


and so $a_{M}=\mu$. As $\lambda>0$, the set $\left\{x \in \Omega: \lambda-b \theta_{[\mu, n]}^{1 / n}(x)>0\right\} \neq \emptyset$ and thus

$$
\theta_{\left[\lambda-b \theta_{[\mu, n]}^{1 / n}, m\right]}>0 \quad \text { for } \mu \geq 0 .
$$

Let

$$
a(x, \mu):=c \theta_{\left[\lambda-b \theta_{[\mu, n]}^{1 / n}, m\right]}^{1 / m}(x) .
$$

¿From Proposition 1, if $\mu_{1} \leq \mu_{2}$ then

$$
a\left(x, \mu_{2}\right) \leq a\left(x, \mu_{1}\right) .
$$

On the other hand, $a(x, 0)=c \theta_{[\lambda, m]}^{1 / m} \gg 0$ and so there exists $\mu_{0}>0$ such that for $0 \leq \mu<\mu_{0}$ we have

$$
0 \leq \mu<\left(a\left(x, \mu_{0}\right)\right)_{M} \leq(a(x, \mu))_{M} .
$$

By the continuity of $a\left(x, \mu_{0}\right)$ there exists $R_{0}>0$ such that for $\mu<\mu_{0}$ the set

$$
\left\{x \in \Omega: \mu \leq a\left(x, \mu_{0}\right)-R_{0}\right\} \neq \emptyset .
$$

Hence,

$$
N(\mu):=N\left(R_{0}, 1\right)=\left\{x \in \Omega: \mu \leq a(x, \mu)-R_{0}\right\} \supset\left\{x \in \Omega: \mu \leq a\left(x, \mu_{0}\right)-R_{0}\right\} \neq \emptyset .
$$

Finally, from (34) it follows easily that if $\mu_{1} \leq \mu_{2}$ then $N\left(\mu_{2}\right) \subset N\left(\mu_{1}\right)$. Now we can reason as in the first part of Theorem 6 .

We will prove the third part. For fixed $\lambda, \mu>0$ and $b>0$, we consider the same function $a(x)$ as above. In this case, we have

$$
N(R, 1):=N(c)=\left\{x \in \Omega: \frac{\mu+R}{c} \leq \theta_{\left[\lambda-b \theta_{[\mu, n]}^{1 / n}, m\right]}^{1 / m}(x)\right\},
$$

and again arguing as for the second part of Theorem 6 the proof follows.

Remark 9 1. Theorem 9 has a biological interpretation similar to Theorem 6 given in Remark 7.

2. The existence of semi-coexistence states of (28) has been studied in [7]. The authors obtained a semi-coexistence state under the condition (32), which is stronger than $\lambda, \mu>0$. It seems that the existence of coexistence states of (32) has not been analyzed previously.

\subsection{Symbiosis}

In this case we consider the cooperative system

$$
\begin{cases}-\Delta u=\lambda u^{1 / m}-u^{2 / m}+b u^{1 / m} v^{1 / n} & \text { in } \Omega, \\ -\Delta v=\mu v^{1 / n}-v^{2 / n}+c v^{1 / n} u^{1 / m} & \text { in } \Omega, \\ u=v=0 & \text { on } \partial \Omega .\end{cases}
$$

Our main result about the existence of semi-coexistence states is the following one: 
Theorem 10 Assume $b c<1$ and that the sets

$$
P_{1}:=\left\{x \in \Omega: \mu+c \theta_{[\lambda, m]}^{1 / m}(x)>0\right\} \neq \emptyset \quad P_{2}:=\left\{x \in \Omega: \lambda+b \theta_{[\mu, n]}^{1 / n}(x)>0\right\} \neq \emptyset .
$$

Then there exists at least one semi-coexistence state of (35).

Remark 10 An observation about the way in which the above conditions should be interpreted is appropriate. If for example $\lambda \leq 0$ then $\theta_{[\lambda, m]}=0$, and so $P_{1} \neq \emptyset$ is equivalent to $\mu>0$.

Proof. Again we use the sub-supersolution method for systems. In this case a couple $(\underline{u}, \bar{u})-(\underline{v}, \bar{v})$ is sub-supersolution of (35) if, see [12], $\underline{u}, \bar{u}, \underline{v}, \bar{v} \in H^{2}(\Omega) \cap L^{\infty}(\Omega), \underline{u} \leq \bar{u}$ and $\underline{v} \leq \bar{v}$ in $\Omega$ and $\underline{u} \leq 0 \leq \bar{u}$ and $\underline{v} \leq 0 \leq \bar{v}$ on $\partial \Omega$ and such that

$$
\begin{cases}-\Delta \underline{u} \leq \lambda \underline{u}^{1 / m}-\underline{u}^{2 / m}+b \underline{u}^{1 / m} \underline{v}^{1 / n} & \text { in } \Omega, \\ -\Delta \bar{u} \geq \lambda \bar{u}^{1 / m}-\bar{u}^{2 / m}+b \bar{u}^{1 / m} \bar{v}^{1 / n} & \text { in } \Omega, \\ -\Delta \underline{v} \leq \mu \underline{v}^{1 / n}-\underline{v}^{2 / n}+c \underline{v}^{1 / n} \underline{u}^{1 / m} & \text { in } \Omega \\ -\Delta \bar{v} \geq \mu \bar{v}^{1 / n}-\bar{v}^{2 / n}+c \bar{v}^{1 / n} \bar{u}^{1 / m} & \text { in } \Omega .\end{cases}
$$

We consider two cases:

1. Assume $\lambda \leq 0$. As we have mentioned above, the condition $P_{1} \neq \emptyset$ is equivalent to $\mu>0$. We take

$$
(\underline{u}, \bar{u})=\left(\theta_{\left[\lambda+b \theta_{[\mu, n]}^{1 / n}, m\right]}, M\right) \quad(\underline{v}, \bar{v})=\left(\theta_{[\mu, n]}, N\right),
$$

with $M, N>0$ constants still to be chosen. Since $P_{2} \neq \emptyset$ then $\underline{u}>0$.

It is easy to show that $M$ and $N$ satisfy (36) provided that

$$
\lambda-M^{1 / m}+b N^{1 / n} \leq 0, \quad \mu-N^{1 / n}+c M^{1 / m} \leq 0 .
$$

Since $b c<1$ there exist $M$ and $N$ satisfying (37). Thus the proof of case 1 is complete.

2. Assume $\lambda>0$. In this case, $P_{2}=\Omega$. Now we pick

$$
(\underline{u}, \bar{u})=\left(\theta_{[\lambda, m]}, M\right) \quad(\underline{v}, \bar{v})=\left(\theta_{\left[\mu+c \theta_{[\lambda, m]}^{1 / m}, n\right]}, N\right),
$$

with $M$ and $N$ as above. This completes the proof.

Again the following results do not need any proof.

Proposition 6 Assume $b c<1$ and let $(u, v)$ be a nonnegative solution of (35). Then

$$
\theta_{[\lambda, m]} \leq u \leq\left(\frac{\lambda+b \mu}{1-b c}\right)^{m} \quad \theta_{[\mu, n]} \leq v \leq\left(\frac{\mu+c \lambda}{1-b c}\right)^{n}
$$

As an immediate consequence of Proposition 6, we have 
Corollary 5 Assume $b c<1$ and either $\lambda+b \mu \leq 0$ or $\mu+c \lambda \leq 0$. Then (35) does not admit a semi-coexistence state.

We obtain the following result on coexistence states

Theorem 11 Assume $b c<1$ and

$$
(\lambda, \mu) \in \overline{\mathbb{R}}_{+} \times \overline{\mathbb{R}}_{+} \backslash\{(0,0)\} .
$$

Then (35) possesses at least one coexistence state.

Proof. Assume $\lambda, \mu>0$. We take

$$
(\underline{u}, \bar{u})=\left(\theta_{[\lambda, m]}, M\right) \quad(\underline{v}, \bar{v})=\left(\theta_{[\mu, n]}, N\right)
$$

with $M$ and $N$ satisfying (37). It is not difficult to show that this couple is a sub-supersolution of (35). On the other hand, if for example $\lambda=0$ and $\mu>0$ we pick

$$
(\underline{u}, \bar{u})=\left(\theta_{\left[b \theta_{[\mu, n]}^{1 / n}, m\right]}, M\right) \quad(\underline{v}, \bar{v})=\left(\theta_{[\mu, n]}, N\right) .
$$

In this case, $M$ and $N$ have to satisfy the following inequalities

$$
-M^{1 / m}+b N^{1 / n} \leq 0, \quad \mu-N^{1 / n}+c M^{1 / m} \leq 0 .
$$

We can reason analogously in the remaining case. This concludes the proof.

The following result can be proved similarly to Proposition 3.

Proposition 7 Assume (39). Then any nonnegative solution of (35) is positive, i.e., it has not a dead core.

The following result says that the set of $(\lambda, \mu) \in \mathbb{R}^{2}$ where (35) has at least a semi-coexistence state is connected.

Theorem 12 Assume $b c<1$. Let us denote

$$
\Gamma:=\left\{(\lambda, \mu) \in \mathbb{R}^{2}:(35) \text { has at least a semi-coexistence state in }(\lambda, \mu)\right\} .
$$

Then $\Gamma$ is connected.

Proof. Let $\left(u_{0}, v_{0}\right)$ be a semi-coexistence state in $\left(\lambda_{0}, \mu_{0}\right)$. We fix $\lambda_{0}$. Let $\mu \in\left[\mu_{0}, \infty\right)$ and define

$$
(\underline{u}, \bar{u})=\left(u_{0}, M\right) \quad(\underline{v}, \bar{v})=\left(v_{0}, N\right)
$$

with $M$ and $N$ satisfying (37). It is not hard to show that this couple is a sub-supersolution of $(35)$ in $\left(\lambda_{0}, \mu\right)$. This completes the proof.

Remark 11 To our knowledge, (35) has been only studied previously in [7]. In this work the authors obtained the existence of a semi-coexistence state under the condition $\lambda>0$ and $\mu>0$. From Theorem 10, (35) possesses a semi-coexistence state even when $\lambda$ or $\mu$ is negative.

Acknowledgements. We are grateful to Professors J. Hernández and J. L. Gámez for helpful comments. The authors thank to DGICYT and CICYT of Spain for research support under grant DGICYT PB95-1242 and MAR98-0486, respectively. 


\section{References}

[1] D. G. Aronson And L. A. Peletier, Large time behaviour of solutions of the porous medium equation in bounded domains, J. Diff. Eqns. 39, 378-412 (1981).

[2] C. Bandle, M. A. Pozio and A. Tesei, The asymptotic behavior of the solutions of degenerate parabolic equations, Trans. A.M.S. 303, 487-501 (1987).

[3] H. Berestycki And P. L. Lions, Some applications of the method of sub and supersolutions, Springer Lectures Notes 782, 16-41 (1980).

[4] J. Blat and K. J. Brown, Bifurcation of steady-state solutions in predator-prey and competition systems, Proc. R. Soc. Edinb., 97A, 21-34 (1984).

[5] H. Brezis ANd L. Oswald, Remarks on sublinear elliptic equations, Nonl. Anal. T.M.A., 10, 55-64 (1986).

[6] R. S. Cantrell and C. Cosner, On the steady-state problem for the Volterra-Lotka competition model with diffusion, Houston J. Math. 13, 337-352 (1987).

[7] A. Cañada and J. L. GÁmez, Elliptic systems with nonlinear diffusion in population dynamics, Diff. Eqns. Dynam. Sys. 3, 189-204 (1995).

[8] C. Cosner And A. C. Lazer, Stable coexistence states in the Volterra-Lotka competition model with diffusion, S.I.A.M. J. Appl. Math. 44, 1112-1132 (1984).

[9] E. N. DAnCer, On positive solutions of some pairs of differential equations, part I, Trans. A.M.S. 284, 729-743 (1984).

[10] E. N. Dancer, On positive solutions of some pairs of differential equations, part II, J. Diff. Eqns. 60, 236-258 (1985).

[11] M. Delgado, J. López-Gómez and A. Suárez, On the symbiotic Lotka-Volterra model with diffusion and transport effects, to appear in J. Diff. Eqns.

[12] M. Delgado And A. SuÁrez, Existence of solutions for elliptic systems with Hölder continuous nonlinearities, to appear in Diff. Int. Eqns.

[13] M. Delgado And A. SuÁrez, On the structure of the positive solutions of the logistic equation with nonlinear diffusion, to appear.

[14] J. I. Díaz And J. HeRnández, On the existence of a free boundary for a class of reaction-diffusion systems, S.I.A.M. J. Math. Anal. 15, 670-685 (1984).

[15] J. C. Eilbeck, J. Furter And J. López-Gómez, Coexistence in the competition model with diffusion, J. Diff Eqns. 107, 96-139 (1994).

[16] M. E. Gurtin and R. C. MacCamy, On the diffusion of biological populations, Math. Biosci. 33, 35-49 (1977).

[17] J. Hernández, Positive solutions for the logistic equation with unbounded limitations, in "Reaction diffusion systems", Lecture Notes in Pure and Applied Mathematics 194, 183-197 (1997).

[18] J. Hernández, Some free boundary problems for predator-prey systems with nonlinear diffusion, Proc. Sympos. Pure Math. 45, 481-488 (1986). 
[19] P. Korman and A. Leung, On the existence and uniqueness of positive steady state in the Volterra-Lotka ecological models with diffusion, Appl. Anal. 26, 145-160 (1987).

[20] A. Leung and G. Fan, Existence of positive solutions for elliptic systems- degenerate and nondegenerate ecological models, J. Math. Anal. Appl. 151, 512-531 (1990).

[21] L. LI, Coexistence theorems of steady states for predator-prey interacting systems, Trans. A.M.S. 305, 143-166 (1988).

[22] L. Li And A. Ghoreishi, On positive solutions of general nonlinear elliptic symbiotic interacting systems, Appl. Anal. 40, 281-295 (1991).

[23] L. Li and R. Logan, Positive solutions to general elliptic competition models, Diff. Int. Eqns. 4, 817-834 (1991).

[24] J. López- Gómez And R. PARdo, Coexistence regions in Lotka-Volterra models with diffusion, Nonl. Anal. T.M.A. 19, 11-28 (1992).

[25] J. LóPEz- Gómez And R. PARdo, Existence and uniqueness of coexistence states for the predatorprey model with diffusion: the scalar case, Diff. Int. Eqns. 6, 1025-1031 (1993).

[26] J. López- Gómez and J. C. SAbina, Coexistence states and global attractivity for some convective diffusive competing species models, Trans. A.M.S 347, 3797-3833 (1995).

[27] Y. Lou, Necessary and sufficient condition for the existence of positive solutions of certain cooperative system, Non. Anal. T.M.A. 26, 1079-1095 (1996).

[28] P. J. McKenna and W. Walter, On the Dirichlet problem for elliptic systems, App. Anal. 21, 207-224 (1986).

[29] M. A. Pozio And A. Tesei, Degenerate parabolic problems in population dynamics, Japan J. Appl. Math. 2, 351-380 (1985).

[30] M. A. Pozio And A. Tesei, Support properties of solutions for a class of degenerate parabolic problems, Comm. Partial Diff. Eqns. 12, 47-75 (1987).

solutions of

[31] Z. Shenghai, Existence of nonnegative solutions for degenerate ecological models, Appl. Math. JCU. 13B, 8-14 (1998). 